\title{
UBIRIS: A Noisy Iris Image Database
}

\author{
Hugo Proença and Luís A. Alexandre \\ Dep. Informatics, Universidade da Beira Interior, \\ IT - Networks and Multimedia Group, Covilhã, \\ R. Marquês D’Ávila e Bolama, 6200-001, Covilhã, Portugal \\ \{hugomcp, 1fbaa\}@di.ubi.pt
}

\begin{abstract}
This paper presents a new iris database that contains images with noise. This is in contrast with the existing databases, that are noise free. UBIRIS is a tool for the development of robust iris recognition algorithms for biometric proposes.

We present a detailed description of the many characteristics of UBIRIS and a comparison of several image segmentation approaches used in the current iris segmentation methods where it is evident their small tolerance to noisy images.
\end{abstract}

\section{Introduction}

The use of biometric systems has been increasingly encouraged by both government and private entities in order to replace or increase traditional security systems.

Iris is commonly recognized as one of the most reliable biometric measures [27]: it has a random morphogenesis and no genetic penetrance [22]. In 1987 L. Flom and A. Safir [9] studied the problem and concluded that iris morphology remains stable through all human life, as well estimated the probability for two similar irises on distinct persons at 1 in $10^{72}$ [27].

In this paper we present UBIRIS [25], a new public and free iris database for biometric proposes. This database has characteristics that clearly distinguish it from the existing ones: CASIA [12] and UPOL [6]. Its main purpose is the evaluation of robust iris identification methodologies. The existing iris databases are noise free: this can be used to teste and develop segmentation and recognition algorithms that are able to work with images captured under near perfect conditions.

The emerging needs for a safer access (buildings, weapons, restricted areas) requires non-invasive, passive techniques. As an example we can think of a building access where the user does not need to look through a small hole to get his iris recognized, but a camera will take a photo (or several) of his iris while he approaches the door. This type of use is much less invasive and will enable the dissemination of iris recognition systems to everyday applications. UBIRIS is a tool for the development of such methods since it exhibits several types of image noise.

We compare the accuracy of several segmentation methodologies to demonstrate their small tolerance to heterogeneous images characteristics, such as the ones exhibited in UBIRIS. 


\subsection{Related Work}

On a general iris recognition system four different stages can be identified: image capture, iris segmentation, feature extraction and feature comparison. In this section we focus on the iris image segmentation.

Since 1987, when the first relevant methodology was presented by Flom and Safir [9], many distinct approaches have been proposed. In 1993, J. Daugman [4] presents one of the most relevant methodologies, constituting the basis of many functioning systems. On the segmentation stage, this author introduces an integrodifferential operator to find both the iris inner and outer borders. This operator remains actual and was proposed with some minor differences in 2004 by [23]. Wildes [26] proposes iris segmentation through a gradient based binary edge map construction followed by circular Hough transform. This methodology is the most widely used, being proposed with minor several variants in [3], [11], [14], [17], [19], [18] and [20].

[16] proposes one simple method based on thresholds and function maximization in order to obtain two ring parameters corresponding to iris inner and outer borders.

Authors from [7] propose one iris detection method based on priori pupil identification. The image is then transformed into polar coordinates and the iris outer border is identified as the largest horizontal edge resultant from Sobel filtering. This approach may fail in case of non-concentric iris and pupil, as well as for very dark iris textures.

Morphologic operators were applied by [21] to obtain iris borders. They detect the inner border by applying threshold, opening and image closing and the outer border with threshold, closing and opening sequence.

Based on the assumption that the image captured intensity values can be well represented by a mixture of three Gaussian distribution components, authors in [13] propose the use of Expectation Maximization [5] algorithm to estimate the respective distributions parameters. They expect that 'Dark', 'Intermediate' and 'Bright' distributions contain the pixels corresponding to the pupil, iris and reflections areas.

Many of described approaches present a major disadvantage: the use of thresholds, usually to construct binary edge maps. This can be considered as a weak point regarding their robustness on image intensity changes.

\section{UBIRIS}

Despite the fact that many of the iris recognition approaches obtain almost optimal results, they do it under particularly favorable conditions, having few image noise factors. These conditions are not easy to obtain and require a high degree of collaboration from the subject, subjecting him to slower and uncomfortable image capture processes.

The aim of UBIRIS is related with this point: it provides images with different types of noise, simulating image captured without or with minimal collaboration from the subjects, pretending to become an effective resource for the evaluation and development of robust iris identification methodologies.

UBIRIS [25] database is composed of 1877 images collected from 241 persons during September, 2004 in two distinct sessions. It constitutes the world's largest public and free available iris database at present date. 


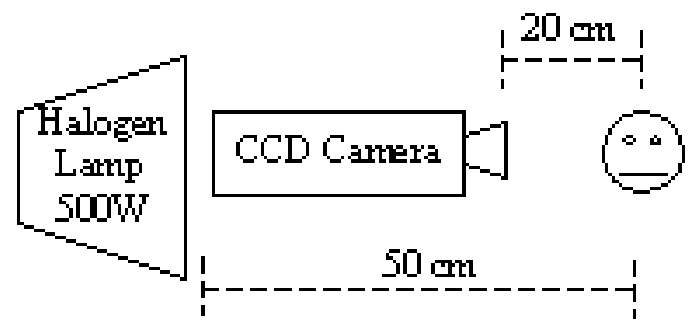

Fig. 1. Image capture framework

We used a Nikon E5700 camera with software version E5700v1.0, 71mm focal length, 4.2 F-Number, 1/30 sec. exposure time, RGB color representation and ISO-200 ISO speed. Images dimensions were 2560x1704 pixels with 300 dpi horizontal and vertical resolution and 24 bit depth. They were saved in JPEG format with lossless compression.

For the first image capture session, the enrollment, we tried to minimize noise factors, specially those relative to reflections, luminosity and contrast, having installed the framework represented in figure 1 inside a dark room.

In the second session we changed the capture location in order to introduce natural luminosity factor. This enabled the appearance of heterogeneous images with respect to reflections, contrast, luminosity and focus problems. Images collected at this stage pretend to simulate the ones captured by a vision system without or with minimal active collaboration from the subjects.

These noisy images will be compared to the ones collected during the enrollment stage.

All images from both sessions are classified with respect to three parameters ('Focus', 'Reflections' and 'Visible Iris') in a three value scale ('Good', 'Average' and 'Bad'). This classification was obtained manually and the results were: focus (Good = $73.83 \%$, Average $=17.53 \%$, Bad $=8.63 \%)$, reflections $($ Good $=58.87 \%$, Average $=$ $36.78 \%, \mathrm{Bad}=4.34 \%)$ and visible iris $($ Good $=36.73 \%$, Average $=47.83 \%$, Bad $=$ $15.44 \%)$

\subsection{PreProcessing}

Preprocessing stage was essential in order to make possible the diffusion of UBIRIS on internet. We reduced the image size to $($ width, height $)=(400,300)$, converted it to grayscale and saved in JPEG format with lossless compression. This process allowed us to obtain images with 27 KBytes average size and still enough quality for the execution of the algorithms.

\section{Segmentation Methodologies}

There are a large number of iris recognition methodologies that present almost optimal results, but only for well-segmented images. The fact that correspondent segmentation methods present much higher error rates, usually above $15 \%$, is overlooked. 
In this section we describe four iris image segmentation methods that are used in the experiments.

Daugman's method. The author [4] assumes both pupil and iris with circular form and applies an integrodifferential operator defined in equation (1).

$$
\max _{r, x_{0}, y_{0}}\left|G_{\sigma}(r) * \frac{\delta}{\delta r} \oint_{r, x_{0}, y_{0}} \frac{I(x, y)}{2 \Pi r} d s\right|
$$

This operator searches over the image domain $(x, y)$ for the maximum in the blurred partial derivative with respect to increasing radius $r$, of the normalized contour integral of $I(x, y)$ along a circular arc $d s$ of radius $r$ and center coordinates $\left(x_{0}, y_{0}\right)$ [4]. In pratical terms, this method searches in $\mathbb{N}^{3}$ for the circumference centre and radius that have the highest derivative value comparing to neighbour radius. This method, as showed in section 4.2, proved to be very effective on images with high intensity separability between iris, pupil and sclera regions. This method was chosen because, although it was proposed about 11 years ago, it remains as one of the most referred on iris segmentation literature and was the first methodology effectively implemented in a working biometric system [8].

Wildes method. Proposed in 1997, this methodology performs iris contour fitting in two steps [26].

First, the image intensity information is converted into a binary edge map. Second, the edge points vote for particular contour parameter values.

The first step is performed via gradient based edge detection [24] [1]. However, before this, the author proposes an histogram based approach to avoid problems with local minima that the active contour model's gradient might experience. Having this, in order to incorporate directional tuning, the image intensity derivatives are weighted to favor ranges of orientation. For example, on the iris/sclera border process, the derivatives are weighted to be selective for vertical edges.

The second step is made through the well known circular Hough transform [10]. This methodology is clearly the most common on iris segmentation approaches, having as principal disadvantage the dependence of threshold values for the edge maps construction. This fact can obviously constitute one weak point as we are concerned with robustness, which includes the ability to deal with heterogeneous image contrast and intensities.

Masek's method. Based on the methodology suggested on [26], this author proposes a method [20] that begins with the binary edge image map construction, using the Kovesi [15] edge detector, a variation of the well known Canny [2] edge detector. The next step consists in applying the circular Hough transform in order to determine the iris/sclera border and then the one correspondent to iris/pupil. This methodology was included on the analysis essentially to represent several approaches on iris segmentation literature. In fact, several other authors propose minor variants to the Wildes [26] method, essentially to adjust the process to different image intensities and contrasts.

Liam and Chekima's method. This iris segmentation approach [16], is based on the fact that the pupil is typically darker than the iris and the iris darker than the sclera. 
Based on this assumption, these authors propose the use of a thresholding technique that converts the initial captured grayscale image to binary. The threshold must be exactly calculated in order to join the pupil and the iris together in a dark region. Assuming that both components have circular form (iris and pupil), the next step consists on creating a ring mask that will run through the whole image searching for the iris/sclera border. The mask radius $r$ and centre coordinates $(x, y)$ will be defined by maximizing equation (2).

$$
S=\sum_{\theta=0}^{2 \Pi}(x+r \cos (\theta), y+r \sin (\theta))
$$

The next step consists in eliminating all image information outside the iris ring, and upgrading the threshold value in order to capture intensity dissimilarities between the iris and the pupil. Pupil/iris border determination is made according to the same methodology described for the iris/sclera border.

As we can see, this method's accuracy is strongly dependent of threshold values that have to be chosen by the user according to captured image characteristics.

\section{Experiments}

In this section we compare results obtained by the methodologies described in section 3 against UBIRIS and CASIA databases.

\subsection{Databases}

The reason for evaluating these methodologies against only the CASIA and UBIRIS databases is related with the fact that UPOL database just includes images from the internal part of the eye, having the segmentation work almost done. Figure 2 shows examples of CASIA database image and the different types of noise that are found on UBIRIS (reflections (2b), focus (2c) and small visible iris part (2d) noise).

\subsection{Results and Discussion}

On table 1 we show the results obtained by each described iris image segmentation method.

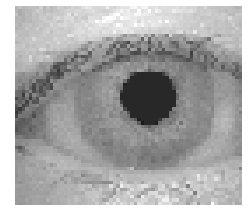

(a) CASIA image database.

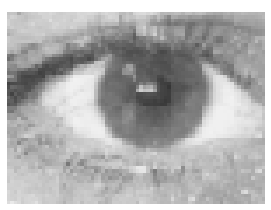

(b) UBIRIS image database (Reflection).

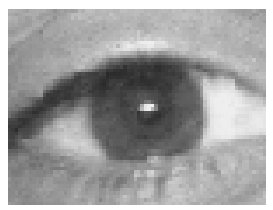

(c) UBIRIS image database (Focus).

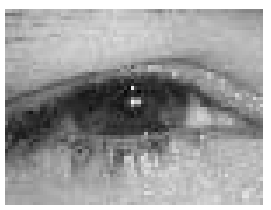

(d) UBIRIS image database (Visible Iris).

Fig. 2. Image examples from two databases used in the experiments 
Table 1. Segmentation accuracy results

\begin{tabular}{|c|c|c|c|c|}
\hline Methodology & Parameters & UBIRIS & CASIA & Notes \\
\hline Daugman & - & $93.53 \%$ & $54.44 \%$ & $\begin{array}{l}\text { Method not dependent from any user } \\
\text { value choice }\end{array}$ \\
\hline Wildes & $\begin{array}{l}\text { Hysteresis Thresholds: Hi=50, Low=44, Gaussian Kernel } \\
\text { Dimension }=5\end{array}$ & $89.12 \%$ & $84.27 \%$ & \begin{tabular}{|l|}
$\begin{array}{l}\text { Parameters optimized for UBIRIS } \\
\text { database }\end{array}$ \\
\end{tabular} \\
\hline Wildes & $\begin{array}{l}\begin{array}{l}\text { Hysteresis Thresholds: Hi=44, Low }=39 \text {, Gaussian Kernel } \\
\text { Dimension= } 5\end{array} \\
\end{array}$ & $81.28 \%$ & $86.49 \%$ & \begin{tabular}{|l|}
$\begin{array}{l}\text { Parameters optimized for CASIA } \\
\text { database }\end{array}$ \\
\end{tabular} \\
\hline Masek & $\begin{array}{l}\text { Gaussian Kernel Dimension=5, Kovesi Parame- } \\
\text { ters }=(40,35)\end{array}$ & $87.12 \%$ & $81.85 \%$ & \begin{tabular}{|l} 
Parameters optimized for UBIRIS \\
database
\end{tabular} \\
\hline Masek & $\begin{array}{l}\text { Gaussian Kernel Dimension=5, Kovesi Parame- } \\
\text { ters }=(39,34)\end{array}$ & $84.16 \%$ & $83.92 \%$ & \begin{tabular}{|l|l|}
$\begin{array}{l}\text { Parameters optimized for CASIA } \\
\text { database }\end{array}$ & \\
\end{tabular} \\
\hline Liam and Chekima & Threshold: 140 & $47.90 \%$ & $56.33 \%$ & \begin{tabular}{|l} 
Parameters optimized for UBIRIS \\
database
\end{tabular} \\
\hline Liam and Chekima & Threshold: 150 & $41.66 \%$ & $64.64 \%$ & \begin{tabular}{|l|l|}
$\begin{array}{l}\text { Parameters optimized for CASIA } \\
\text { database }\end{array}$ & \\
\end{tabular} \\
\hline
\end{tabular}

All evaluated methods presented distinct accuracy levels on each database. This fact indicates that their accuracy is clearly dependent of the image characteristics, adding one relevant restriction to respective efficiency.

Daugman's [4] methodology, despite not being based on thresholds, presented the most heterogeneous results, having the best and the worst accuracy respectively on UBIRIS and CASIA databases. This fact can be easily explained by the lower contrast between iris and sclera eye parts on CASIA images. Trying to identify the circumference parameters (centre and radius) where the average intensity values have maximum derivative in respect to neighbour ones tends to identify circumferences tangent to pupil region. UBIRIS images have greater contrast between iris, pupil and sclera parts, thus yielding a better accuracy.

Approaches proposed by Wildes [26] and Masek [20] are similar on their methodology, therefore on their results, and have presented a more robustness behavior. However both methods are based on thresholds for constructing binary edges maps. This is an obvious disadvantage comparing to other image characteristics.

Apart from being the less accurate, thus obtaining worst results, methodology proposed by Liam and Chekima[16] was the less tolerant to image characteristic changes. This fact can be easily explained by the important role of the threshold operator that is the basis for both inner and outer border iris detection. In particular, probably motivated by the UBIRIS images characteristics, this methodology didn't at any circumstance reach the $50 \%$ accuracy, as opposite to CASIA database where accuracy was beyond $64 \%$.

\section{Conclusions}

We presented a new public and free iris database for biometric proposes and described the most important aspects that distinguish it from the existing ones. 
We encourage the use of this database by anyone who works or has interest on the area. It is available on http: / / iris.di.ubi.pt and can be downloaded with noise classification statistics.

We stress the minor importance that some authors give to segmentation stage, assuming that it is a trivial and error-free stage. Section 4.2 clearly showed that the selected methods deteriorate their performance in direct proportion with changes in image quality and characteristics.

Present work concerns the exhaustive test of existing iris image segmentation methodologies against available databases trying to identify and propose a more robust one.

\section{Acknowledgements}

We would like to thank all the persons that gave their contribute on UBIRIS images capture. In particular, we had the support from the 'Optics Center' and 'Multimedia Center' from Universidade da Beira Interior.

\section{References}

1. D. H. Ballard and C. M. Brown. Computer Vision. Englewood Cliffs, NJ: Prentice-Hall, E.U.A., 1982.

2. J. Canny. A computational approach to edge detection. In IEEE Trans. on Pattern Analysis and Machine Intelligence, vol. 8, pages 679-698.

3. Jiali Cui, Yunhong Wang, Tieniu Tan, Li Ma, and Zhenan Sun. A fast and robust iris localization method based on texture segmentation. 2004. http://nlpr-web.ia.ac.cn/english/irds/papers/cuijl/SPIE.pdf

4. John G. Daugman. High confidence visual recognition of persons by a teste of statistical independence. In IEEE Transactions on Pattern Analysis and Machine Intelligence, vol. 15, no. 11, pages 1148-1161, U.S.A., 1993.

5. A. P. Dempster, N. Laird, and D. Rubin. Maximum likelyhood from incomplete data via the em algorithm. In Journal of the Royal Statistic Society, vol. 39, pages 1-38, 1977.

6. Michal Dobes and Libor Machala. Upol iris image database. 2004. http://phoenix.inf.upol.cz/iris/

7. Yngzi Du, Robert Ives, Delores Etter, Thad Welch, and Chein Chang. A new approach to iris pattern recognition. In SPIE European Symposium on Optics/Photonics in Defence and Security, 2004.

8. Jacqueline Emigh. The eyes have it. 2003. http: / / securitysolutions.com/mag/security_eyes /

9. Leonard Floom and Aran Safir. Iris recognition system, u.s. patent no. 4.641.349. 1987.

10. P. V. C. Hough. Method and means for recognizing complex patterns. 1962. U.S. Patent 3 069654.

11. Junzhou Huang, Yunhong Wang, Tieniu Tan, and Jiali Cui. A new iris segmentation method for recognition. In Proceedings of the 17th International Conference on Pattern Recognition (ICPR04), 2004.

12. Chinese Academy of Sciences Institute of Automation. Casia iris image database. 2004. http: / /www.sinobiometrics.com 
13. Jaemin Kim, Seongwon Cho, and Jinsu Choi. Iris recognition using wavelet features. In Kluwer Academic Publishers, Journal of VLSI Signal Processing, no. 38, pages 147-156, The Netherlands, 2004.

14. W. K Kong and D. Zhang. Accurate iris segmentation method based on novel reflection and eyelash detection model. In Proceedings of 2001 International Symposium on Inteligent Multimedia, Video and Speech Processing, Hong Kong, May 2001.

15. Peter Kovesi. Matlab functions for computer vision and image analysis. 2004. http: / / cs. uwa. edu.au/ pk/Research/MatlabFns /

16. Lye Liam, Ali Chekima, Liau Fan, and Jamal dargham. Iris recognition using self-organizing neural network. In IEEE, 2002 Student Confderence on Research and Developing Systems, pages 169-172, Malasya, 2002.

17. Li Ma, Tieniu Tan, Yunhong Wang, and Dexin Zhang. Personal identification based on iris texture analysis. In IEEE Transactions on Pattern Analysis and Machine Intelligence, vol. 25, no. 12, pages 1519-1533, U.S.A., 2003.

18. Li Ma, Yunhong Wang, and Tieniu Tan. Iris recognition based on multichannel gabor filtering. In ACCV2002: The 5th Asian Conference on Computer Vision, Australia, 2002.

19. Li Ma, Yunhong Wang, and Dexin Zhang. Efficient iris recognition by characterizing key local variations. In IEEE Transactions on Image Processing, vol. 13, no. 6, pages 739-750, U.S.A., 2004.

20. Libor Masek. Recognition of human iris patterns for biometric identification. 2003. http://www.csse.uwa. edu. au/ pk/studentprojects/libor

21. J. Mira and J. Mayer. Image feature extraction for application of biometric identification of iris - a morphological approach. In IEEE Proceedings of the XVI Brazilian Symposium on Computer Graphics and Image Processing (SIBGRAPIO3), 2003.

22. Alan Muron and Jaroslav Pospisil. The human iris structure and its usages. In Acta Univ. Palacki, Phisica, vol. 39, pages 87-95, 2000.

23. Ko Nishino and Shree K. Nayar. Eyes for relighting. ACM Trans. Graph., 23(3):704-711, 2004.

24. W. K. Pratt. Digital Image Processing. Wiley, U.S.A., 1978.

25. Hugo Proença and Luís A. Alexandre. Ubiris iris image database. 2004. http: //iris.di.ubi.pt

26. Richard P. Wildes. Iris recognition: an emerging biometric technology. In Proceedings of the IEEE, vol. 85, no.9, pages 1348-1363, U.S.A., 1997.

27. Gerald Williams. Iris recognition technology, iridian technologies, inc. 2001. http: / /www. argus-solutions.com/pdfs/irisrecogwilliams.pdf 